\title{
Catheter-related Bacteremia Caused by Agrobacterium radiobacter in a Hemodialysis Patient
}

\author{
Shigeru Hanada ${ }^{1}$, Mari Iwamoto ${ }^{1}$, Namiko Kobayashi ${ }^{1}$, Ryoichi Ando ${ }^{1}$ and Sei Sasaki ${ }^{2}$
}

\begin{abstract}
Agrobacterium radiobacter, a Gram-negative bacillus, is recognized as an emerging opportunistic human pathogen that has a propensity to cause infections in patients with indwelling foreign devices. Here, we describe the first reported case of catheter-related bacteremia caused by $A$. radiobacter in a hemodialysis patient with a long-term tunneled-cuffed hemodialysis catheter. This case shows that A. radiobacter should be included in the list of pathogens that can cause catheter-related bacteremia in hemodialysis patients.
\end{abstract}

Key words: Agrobacterium radiobacter, catheter-related bacteremia, hemodialysis, tunneled-cuffed catheter

(Inter Med 48: 455-457, 2009)

(DOI: 10.2169/internalmedicine.48.1754)

\section{Introduction}

The number of hemodialysis patients with a tunneledcuffed hemodialysis catheter (TCHC) for long-term vascular access has increased in recent years, due to an increase in patients in whom construction of an arteriovenous fistula or graft is difficult because of exhausted vasculature or severely impaired cardiac function. Catheter-related bacteremia (CRB) is a serious complication of a TCHC that can lead to substantial morbidity and catheter removal, and may occasionally be fatal (1).

The causative organisms of CRB are Gram-positive cocci (Staphylococcus and Enterococcus species) in approximately $60 \%$ of hemodialysis patients with TCHCs, with a wide variety of Gram-negative bacilli accounting for about 30\% of cases. Mixed infections and fungal organisms make up the remaining isolates $(1,2)$. The incidence of $\mathrm{CRB}$ due to Gram-negative bacilli in hemodialysis patients is increasing, reflecting trends in isolate patterns reported for patients with non-dialysis central venous catheters (3), and unusual organisms such as Serratia, Acinetobacter and Citrobacter are also emerging as opportunistic pathogens $(1,2)$. Here, we describe a unique case of CRB caused by one such uncommon Gram-negative bacillus, Agrobacterium radiobacter, in a patient receiving chronic hemodialysis with a TCHC.

\section{Case Report}

The patient was a 75-year-old Japanese woman who had received hemodialysis 3 times a week for 3 years due to end-stage kidney disease secondary to diabetic nephropathy. She was referred from an outpatient hemodialysis unit because of spiking fever with chills after hemodialysis sessions over 2 weeks. The hemodialysis was performed via a right subclavian TCHC (Soft-Cell ${ }^{\mathrm{TM}}$; C.R. Bard., $12.5 \mathrm{Fr}, 19 \mathrm{~cm}$ ) that had been inserted 2 years earlier for long-term vascular access, since she had no other options for vascular access due to markedly decreased cardiac function (LVEF 28\%) caused by severe coronary disease and exhausted vasculature.

A physical examination failed to reveal a sign of infection, including in the tunnel tract and exit-site of the TCHC. The patient had severe symptoms of heart failure and showed marked weakening. Blood pressure was 84/38 $\mathrm{mmHg}$, heart rate was 70 beats/min and regular, and body temperature was $36.6^{\circ} \mathrm{C}$ (18 hours after the hemodialysis session). Laboratory tests revealed a white blood cell (WBC) count of $6.2 \times 10^{3} / \mu \mathrm{L}\left(6.2 \times 10^{9} / \mathrm{L}\right)$ and a C-reactive protein (CRP) level of $6.44 \mathrm{mg} / \mathrm{dL}$. All other data were unremarkable. A chest X-ray showed mild congestive heart failure. Gallium scintigraphy and computed tomography of

${ }^{1}$ Department of Nephrology, Musashino Red Cross Hospital, Tokyo and ${ }^{2}$ Department of Nephrology, Graduate School of Medicine, Tokyo Medical and Dental University, Tokyo

Received for publication October 7, 2008; Accepted for publication December 2, 2008

Correspondence to Dr. Shigeru Hanada, s-hanada@umin.ac.jp 
the brain, chest and abdomen showed no evidence of infection or malignancy.

Empirical antibiotic therapy with parenteral ampicillin/sulbactam (1.5 g/day) was initiated, based on a suspected diagnosis of CRB from the symptom of spiking fever after the hemodialysis session. Two sets of blood cultures using samples from peripheral veins and one set based on samples taken through the lumen of the TCHC were obtained. All three sets yielded a Gram-negative bacillus, which was later identified as $A$. radiobacter. Therefore, the patient was diagnosed with $\mathrm{CRB}$ caused by $A$. radiobacter. The isolates were susceptible to ampicillin, cefotiam, cefmetazole, imipenem/cilastatin, meropenem, amikasin, minomycin and levofloxacin, but resistant to piperacillin, fosfomycin and ceftazidime.

The patient showed rapid abatement of fever, remained afebrile and showed clinical improvement. Thus, we continued to attempt to salvage the TCHC as long as the patient had a good clinical response to antibiotic therapy. Antibiotics were administered for 21 days and the infection seemed to have been resolved completely. However, spiking fever of $39^{\circ} \mathrm{C}$ developed again after a hemodialysis session 3 days after completion of antibiotic therapy. The same antibiotic therapy was reinitiated and two sets of blood cultures from peripheral veins both yielded $A$. radiobacter. In view of the relapsing nature of $\mathrm{CRB}$, we decided to remove the catheter since we judged that salvage of the TCHC would be difficult. The period from TCHC insertion to removal was 736 days. Cultures of the cuff of the catheter and the catheter tip gave negative findings. The infection was controlled efficiently after removal of the TCHC, but the patient had difficulty with hemodialysis due to further exacerbation of cardiac function and died of heart failure 4 weeks after removal of the catheter. The death was not directly attributed to CRB.

\section{Discussion}

Members of the genus Agrobacterium are aerobic, motile, oxidase-positive, and non-spore-forming Gram-negative bacilli. These organisms are found chiefly in soils worldwide and are primarily known to cause tumorigenic plant disease. $A$. radiobacter is the most common species of the genus known to cause human disease. This organism is found only infrequently in clinical specimens, but is emerging as a rare opportunistic pathogen that causes infections in chronically debilitated patients. A. radiobacter infection can be resolved in the majority of patients and is rarely fatal, indicating that this organism has low virulence (4).

Mastroianni et al (5) summarized 34 cases of A. radiobacter infections from 1980 to 1996, and thereafter Paphitou and Rolston (6) described 8 additional cases from 1996 to 2002. Of the combined 42 cases, $81 \%$ (34/42) were associated with the presence of foreign devices (e.g., indwelling intravascular devices, peritoneal dialysis catheters, nephrostomy tubes) and 93\% (39/42) had underlying conditions in- cluding an immunocompromised state (cancer, hematologic malignancy, AIDS) or debilitation due to a chronic disease such as end-stage kidney disease requiring peritoneal dialysis. These data indicate that $A$. radiobacter is an opportunistic organism that has the propensity to cause infection in patients with indwelling foreign devices. CRB, peritonitis that is usually associated with peritoneal dialysis, urinary infections in the presence of nephrostomy tubes, and pneumonia are the most common clinical conditions associated with $A$. radiobacter infection (5-7). The strong correlation between A. radiobacter and indwelling foreign devices may be explained by the finding of Alnor et al that this organism showed marked adhesion to silicone tubes, comparable to Staphylococcus species, in an experimental model (8).

The mechanism of acquisition of $A$. radiobacter has yet to become clear. Although the organism is found in soil, only one case report has mentioned probable central venous catheter contamination from exposure to soil before development of $A$. radiobacter infection (9). The patient in the current case was not exposed to soil or plant material as an explanation for inoculation, and the source of bacteria and the route of bacterial entry to the bloodstream are unknown.

Diagnosis of CRB in a hemodialysis patient is definite when the same organism is cultured from the catheter tip and from a peripheral or catheter blood sample in a symptomatic patient with no other apparent source of infection. The catheter tip culture gave a negative finding in this case, but we believe that CRB caused by $A$. radiobacter was the appropriate diagnosis because two samples from peripheral blood and a sample from catheter blood all yielded A. radiobacter without another an evident source and the infection was efficiently controlled after removal of the TCHC. In addition, spiking fever after hemodialysis indicated that bacteria or pyrogens sequestered in or around the TCHC had been released by blood flow through the TCHC. The negative finding for the catheter tip culture may have reflected the use of antibiotics delivered through the TCHC.

This case showed typical characteristics of A. radiobacter infection, since the patient had an indwelling intravascular device and was a chronically debilitated host due to underlying end-stage kidney disease, impaired cardiac function, and diabetes mellitus. We believe that this is the first reported case of CRB caused by $A$. radiobacter in a hemodialysis patient. The case indicates that $A$. radiobacter should now be viewed as an emerging pathogen of opportunistic infections in hemodialysis patients, particularly given the increasing number of patients with indwelling foreign devices who are chronically debilitated due to diabetes mellitus, cardiovascular disease or complications related to chronic hemodialysis.

The clinical practice guidelines for vascular access published by the Kidney Disease Outcomes Quality Initiative of the National Kidney Foundation (NKF-K/DOQI) $(10,11)$ indicate that the TCHC should be removed in a clinically unstable CRB patient regardless of the causal organism. In addition, these guidelines recommend treatment of stable CRB patients without exit-site or catheter tunnel tract in- 
volvement who become afebrile after initial antibiotic therapy by exchanging the catheter over a guidewire plus a minimum of 3 weeks of systemic antibiotics or catheter salvage with systemic antibiotics in conjunction with antibiotic locks. Parenteral antibiotic therapy alone is not recommended due to its low success rate $(25-32 \%)(1,2,12)$. However, a lack of clinical evidence makes it unclear whether these guidelines are applicable in cases caused by A. radiobacter.

There is no consensus regarding the need for removal of indwelling foreign devices in treatment of A. radiobacter infections. The guidelines for management of intravascular catheter-related infections published by the Infectious Diseases Society of America (IDSA) (13) lists the Agrobacterium species among the Gram-negative organisms for which "serious consideration should be given to catheter removal, especially if bacteremia continues despite appropriate antibiotics or if the patient becomes unstable." Of the combined
42 cases described above, $48 \%$ of CRB cases (10/21) were treated successfully without catheter removal and this success rate was higher than that of other organisms. Therefore, we initially attempted to salvage the TCHC based on the prior evidence of an effect of antibiotic therapy.

Based on literature data and our experience, antibiotic therapy for salvage of an intravascular catheter in CRB caused by A. radiobacter is a good initial choice, but caution must be exercised since the infection can relapse after therapy, even in cases in which the antibiotic therapy appears to have sufficiently suppressed $A$. radiobacter, as in the current case. The catheter should be removed immediately for a better outcome upon relapse of the infection. In conclusion, we suggest that $A$. radiobacter should be included in the list of pathogens that cause CRB in hemodialysis patients with a TCHC, and that the course of treatment should be determined on a case-by-case basis and may involve catheter removal.

\section{References}

1. Marr KA, Sexton DJ, Conlon PJ, Corey GR, Schwab SJ, Kirkland KB. Catheter-related bacteremia and outcome of attempted catheter salvage in patients undergoing hemodialysis. Ann Intern Med 127: 275-280, 1997.

2. Saad TF. Bacteremia associated with tunneled, cuffed hemodialysis catheters. Am J Kidney Dis 34: 1114-1124, 1999.

3. Velasco E, Byington R, Martins CS, Schirmer M, Dias LC, Gonçalves VM. Bloodstream infection surveillance in a cancer centre: a prospective look at clinical microbiology aspects. Clin Microbiol Infect 10: 542-549, 2004.

4. Edmond MB, Riddler SA, Baxter CM, Wicklund BM, Pasculle AW. Agrobacterium radiobacter: a recently recognized opportunistic pathogen. Clin Infect Dis 16: 388-391, 1993.

5. Mastroianni A, Coronado O, Nanetti A, Manfredi R, Chiodo F. Agrobacterium radiobacter pneumonia in a patient with HIV infection. Eur J Clin Microbiol Infect Dis 15: 960-963, 1996.

6. Paphitou NI, Rolston KV. Catheter-related bacteremia caused by Agrobacterium radiobacter in a cancer patient: case report and literature review. Infection 31: 421-424, 2003.

7. Lai CC, Teng LJ, Hsueh PR, et al. Clinical and microbiological characteristics of Rhizobium radiobacter infections. Clin Infect Dis 38: 149-153, 2004.
8. Alnor D, Frimodt-Møller N, Espersen F, Frederiksen W. Infections with the unusual human pathogens Agrobacterium species and Ochrobactrum anthropi. Clin Infect Dis 18: 914-920, 1994.

9. Chen CY, Hansen KS, Hansen LK. Rhizobium radiobacter as an opportunistic pathogen in central venous catheter-associated bloodstream infection: case report and review. J Hosp Infect 68: 203-207, 2008.

10. National Kidney Foundation, Dialysis Outcomes Quality Initiative. NKF-DOQI Clinical Practice Guidelines for Vascular Access: update 2000. Am J Kidney Dis 37 (Suppl 1): S137-S181, 2001.

11. Vascular Access, Work Group. Clinical practice guidelines for vascular access. Am J Kidney Dis 48 (Suppl 1): S248-S273, 2006.

12. Moss AH, Vasilakis C, Holley JL, Foulks CJ, Pillai K, McDowell DE. Use of a silicone dual-lumen catheter with a Dacron cuff as a long-term vascular access for hemodialysis patients. Am J Kidney Dis 16: 211-215, 1990.

13. Mermel LA, Farr BM, Sherertz RJ, et al. Infectious Diseases Society of America; American College of Critical Care Medicine; Society for Healthcare Epidemiology of America. Guidelines for the management of intravascular catheter-related infections. Clin Infect Dis 32: 1249-1272, 2001.

(C) 2009 The Japanese Society of Internal Medicine http://www.naika.or.jp/imindex.html 